\title{
SUR UNE CLASSE DE FLUIDES NON NEWTONIENS: LES SOLUTIONS AQUEUSES DE POLYMĖRES
}

\author{
BY \\ C. AMROUCHE \\ Université Pierre et Marie Curie, Paris, France
}

\begin{abstract}
Resumé. L'objet de ce travail est d'étudier un système d'évolution non linéaire de troisième ordre représentant une approximation des équations de Navier-Stokes. Ce système permet de décrire le mouvement de fluides visqueux auxquels on a ajouté une petite substance de polymères, ce qui a pour effet de changer fondamentalement les propriétés de relaxation du fluide résultant.

On se place ici dans le cas d'un ouvert borné suffisamment régulier de $\mathbb{R}^{n}, n=2$ ou 3, et on montre l'existence et l'unicité d'une solution locale en temps pour des données suffisamment régulières (la vitesse initiale $u_{0} \in H^{3}$, les forces extérieures $\left.f \in L^{2}\left(0, T ; H^{1}\right)\right)$, cela grâce à l'utilisation d'une base spéciale appropriée (cf. D. Cioranescu et E. H. Ouazar) dans la méthode de Galerkin. On donne ensuite un critère d'unicité dans une classe où l'existence n'est cependant pas assurée.
\end{abstract}

Abstract. The aim of this paper is to study a nonlinear evolution system of third order representing an approximation of Navier-Stokes equations. This system describes the motion of a viscous fluid to which a small quantity of polymers is added. The consequently main relaxation properties of the resulting fluid are completely changed.

We consider the case of a smooth open bounded set of $\mathbb{R}^{n}, n=2$ or 3 , and show the existence and the uniqueness of a solution, local in time, for sufficiently regular data (the initial velocity $u_{0} \in H^{3}$, the external force $\left.f \in L^{2}\left(0, T ; H^{1}\right)\right)$. The basic tool is a suitable special basis to be used in the Galerkin method. In a special class of functions, we give a uniqueness criterium (but where the existence is not ensured).

1. Introduction. Il a été constaté expérimentalement que si l'on ajoute une petite quantité d'une substance de polymères dans un fluide visqueux en mouvement, sans changement de densité ni de viscosité, il apparaît alors un phénomène de couches limites (cf. [7]). Une comparaison des propriétés physiques de l'eau et d'une solution aqueuse de polymères montre que, pour des valeurs très proches de densité et de viscosité, ces deux types de fluides possèdent des propriétés de relaxation fondamentalement différentes (le processus de relaxation du premier étant beaucoup plus rapide par rapport au second).

Received February 13, 1991.

(C)1992 Brown University 
La loi de Newton

$$
T=-p I+2 \nu D
$$

où $T$ est le tenseur des contraintes, $D$ le tenseur des taux de déformation, $D(u)=$ $\frac{1}{2}\left(\partial_{i} u_{j}+\partial_{j} u_{i}\right), p$ la pression, $I$ la matrice identité, et $\nu$ la viscosité, n'est pas une loi générale permettant de décrire le comportement de tous les fluides. Elle est notamment obtenue sous l'hypothèse que le fluide ne possède pas de propriétés de relaxation. Afin de prendre en considération ces propriétés, il a été suggéré (cf. [7]) de prendre comme loi constitutive

$$
T=-p I+2 \nu D+2 \alpha d / d t(D),
$$

où $\alpha$ est le coefficient de relaxation et la dérivée particulaire donnée par

$$
d / d t=\partial / \partial t+u \cdot \nabla
$$

$u=\left(u_{i}\right)$ désignant le vecteur champ de vitesses.

Par ailleurs, pour que l'énergie libre de Helmhotz soit au minimum à l'équilibre, il est nécessaire de prendre (cf. [1]) $\alpha$ tel que

$$
\alpha>0 \text {. }
$$

On s'intéresse aux fluides incompressibles occupant une région bornée, $n=2$ ou 3 , avec comme conditions aux limites l'adhérence à la frontière. Sous cette condition et compte-tenu des équations de mouvement, un fluide incompressible satisfaisant à la loi (1.2) vérifie

$$
\begin{gathered}
u^{\prime}-\alpha \Delta u^{\prime}-\nu \Delta u+(u \cdot \nabla)(u-\alpha \Delta u)+\nabla p=f \quad \text { dans } Q_{T}, \\
\operatorname{div} u=0 \quad \operatorname{dans} Q_{T}, \\
u_{\mid \Gamma}=0, \quad t \in(0, T), \\
u(0)=u_{0} \quad \operatorname{dans} \Omega,
\end{gathered}
$$

où $u^{\prime}=\partial u / \partial t, f$ désigne les forces extérieures, $u_{0}$ la vitesse initiale, et $Q_{T}=$ $(0, T) \times \Omega, T>0$.

Remarque 1.1. Pour $\alpha=0$, on retrouve les équations de Navier-Stokes.

Remarque 1.2. Dans le cas des fluides de grade 2, l'équation (1.5) est remplacée par

$$
u^{\prime}-\alpha \Delta u^{\prime}-\nu \Delta u+\operatorname{rot}(u-\alpha \Delta u) \wedge u+\nabla p=f \text { dans } Q_{T} .
$$

Dans une étude faite par D. Cioranescu et E. H. Ouazar [3], ces auteurs ont établi l'existence de solutions pour le système (1.6)-(1.9). Mais on remarque que

$$
\operatorname{rot}(u-\alpha \Delta u)=(u \cdot \nabla)(u-\alpha \Delta u)-\sum_{j=1}^{n} u_{j} \nabla\left(u_{j}-\alpha \Delta u_{j}\right),
$$

de sorte qu'il est raisonnable d'espérer obtenir ici des résultats similaires à ceux des fluides de grade 2 .

Remarque 1.3. Le système (1.5)-(1.8) a déjà été étudié par A. P. Oskolkov $[7,8]$. Dans une de ses démonstations, celui-ci utilise une base spéciale qui ne me semble pas fournir l'outil adéquat permettant de trouver par la méthode de Galerkin 
des solutions approchées du problème. Celle-ci ne vérifie en effet pas la condition d'incompressibilité (1.6).

Le paragraphe 2 est consacré aux espaces fonctionnels et à la formulation variationnelle du problème. Dans le paragraphe 3 , on établit un résultat d'existence et d'unicité de la solution dans les cas bi- et tri-dimensionnel. Une autre formulation du problème est donnée dans le dernier paragraphe.

2. Espaces fonctionnels et formulation variationnelle. Soit $\Omega$ un ouvert borné de $\mathbb{R}^{n}, n=2$ ou 3 , de frontière $\Gamma$ suffisamment régulière. On pose

$$
\begin{aligned}
& \mathscr{V}=\left\{v \in[\mathscr{D}(\Omega)]^{n} ; \operatorname{div} v=0\right\}, \\
& H=\text { adhérence de } \mathscr{V} \text { dans }\left[L^{2}(\Omega)\right]^{n}, \\
& \left.V=\text { adhérence de } \mathscr{V} \text { dans }\left[H^{1} \Omega\right)\right]^{n}, \\
& W=V \cap\left[H^{2}(\Omega)\right]^{n} .
\end{aligned}
$$

L'espace $H$ est muni du produit scalaire et de la norme usuels de $\left[L^{2}(\Omega)\right]^{n}$

$$
(u, v)=\int_{\Omega} u_{i} v_{i} d x, \quad|v|=(v, v)^{1 / 2} \quad \forall u, v \in H
$$

(on adoptera pour toute la suite la convention de sommation explicite sur les indices répétés).

Pour $u, v \in V$, on pose

$$
((u, v))=\int_{\Omega} \nabla u \cdot \nabla v d x
$$

Muni du produit scalaire

$$
(u, v)_{V}=(u, v)+\alpha((u, v)),
$$

l'espace $V$ est un Hilbert; sa norme correspondante

$$
|v|_{1}=\left(|v|^{2}+\alpha\|v\|^{2}\right)^{1 / 2}, \quad \alpha>0 .
$$

De même, $W$ est un Hilbert pour le produit scalaire

$$
\begin{aligned}
(u, v)_{W} & =(u-\alpha \Delta u, v-\alpha \Delta v) \\
& =(u, v)+2 \alpha((u, v))+\alpha^{2}(\Delta u, \Delta v) \quad \forall u, v \in W .
\end{aligned}
$$

La norme correspondante, équivalente à celle de $\left[H^{2}(\Omega)\right]^{n}$, sera notée $|v|_{2}=$ $|v-\alpha \Delta v|$. On rapelle que (cf. [9])

$$
\begin{aligned}
H & =\left\{v \in\left[L^{2}(\Omega)\right]^{n} ; \operatorname{div} v=0,\left.v \cdot n\right|_{\Gamma}=0\right\}, \\
V & =\left\{v \in\left[H_{0}^{1}(\Omega)\right]^{n} ; \operatorname{div} v=0\right\} ;
\end{aligned}
$$

et si l'on identifie $H$ à son dual $H^{\prime}$, alors $W=D(A)$ où $D(A)$ est le domaine de l'opérateur $A \in \mathscr{L}\left(V ; V^{\prime}\right)$ défini par

$$
\langle A(u), v\rangle_{V^{\prime}, V^{\prime}}=((u, v)) \quad \forall u, v \in V .
$$

On définit également la forme trilinéaire suivante $b$ par

$$
b(u, v, w)=\int_{\Omega} u_{i} \partial_{i} v_{j} w_{j} d x
$$


qui est continue sur $V \times V \times V$ et satisfait (cf. [6], [5], ou [9])

$$
b(u, v, w)=-b(u, w, v) \quad \forall u \in V, v, w \in\left[H^{1}(\Omega)\right]^{n} .
$$

De plus (cf. [2]),

$$
b(v, u, \Delta u)=b(\Delta u, u, v) \quad \forall u \in W, v \in V \text { si } n=2 .
$$

Avec ces notations, une formulation faible du problème (1.5)-(1.8) est

$$
\begin{gathered}
\text { trouver } u(t) \in V \cap\left[H^{3}(\Omega)\right]^{n}, \\
\left(u^{\prime}, v\right)_{V}+\nu((u, v))-b(u, v, u-\alpha \Delta u)=(f, v) \quad \forall v \in V \cap\left[H^{3}(\Omega)\right]^{n}, \\
u(0)=u_{0} .
\end{gathered}
$$

Remarquons que la forme $b(u, v, w-\alpha \Delta w)$ est continue sur $V \times W \times W$.

On introduit maintenant les espaces fonctionnels

$$
\begin{aligned}
& V_{3}=\left\{v \in\left[H^{3}(\Omega)\right]^{n} ; \operatorname{div} v=0,\left.v\right|_{\Gamma}=0\right\}, \\
& \widetilde{V}_{3}=\left\{v \in\left[H_{0}^{1}(\Omega)\right]^{n} ; \operatorname{div} v=0, \operatorname{rot}(v-\alpha \Delta v) \in\left[L^{2}(\Omega)\right]^{n}\right\} .
\end{aligned}
$$

On rappelle le résultat suivant dû à $D$. Cioranescu et E. H. Ouazar [3].

THÉORÈME 2.1. Si $\Gamma$ est suffisamment régulière, on a les identités algèbriques et topologiques $V_{3}=\widetilde{V}_{3}$, et il existe une constante $C>0$, dépendant seulement de $\Omega$, telle que

$$
|v|_{3} \leq C\left(|v|_{1}^{2}+|\operatorname{rot}(v-\alpha \Delta v)|^{2}\right)^{1 / 2} \quad \forall v \in V_{3},
$$

où $|\cdot|_{3}$ désigne la norme dans $\left[H^{3}(\Omega)\right]^{n}$.

Comme conséquence de ce théorème, l'espace $V_{3}$ muni du produit scalaire

$$
(u, v)_{V_{3}}=(u, v)_{V}+(\operatorname{rot}(u-\alpha \Delta u), \operatorname{rot}(v-\alpha \Delta v)) \quad \forall u, v \in V_{3}
$$

est un espace de Hilbert.

Dans toute la suite on identifiera $V$ à son dual $V^{\prime}$; on a alors les injections

$$
V_{3} \subset W \subset V \subset W^{\prime} \subset V_{3}^{\prime},
$$

où chaque espace est dense dans le suivant et les injections sont continues. De plus, les injections $V_{3} \subset W \subset V$ sont compactes.

RemarQue 2.1. Dans la théorie des équations de Navier-Stokes, on travaille avec les espaces $V$ et $H$ et les injections $V \subset H=H^{\prime} \subset V^{\prime}$. Ici l'espace pivot $H$ est remplacé par $V$; et c'est ce même choix qui va nous permettre, comme pour les fluides de grade 2 et de grade 3, d'obtenir des résultats d'existence de solutions du problème (2.3)-(2.4).

Proposition 2.2 (Base spéciale). Comme l'injection $V_{3} \subset V$ est compacte, le problème spectral

$$
(w, v)_{V_{3}}=\lambda(w, v)_{V} \quad \forall v \in V_{3},
$$

admet une suite de solutions (fonctions propres) $\left\{w_{j}\right\}$ correspondant à une suite de valeurs propres $\left\{\lambda_{j}\right\}$

$$
\left(w_{j}, v\right)_{V_{3}}=\lambda_{j}\left(w_{j}, v\right)_{V} \quad \forall v \in V_{3}
$$


avec $0<\lambda_{1}<\cdots<\lambda_{k}<\cdots \rightarrow+\infty$. Les fonctions $w_{j}$ forment une base orthonormale dans $V$ et orthogonale dans $V_{3}$.

C'est cette suite de fonctions qu'on utilisera plus loin comme base spéciale dans la méthode de Galerkin.

3. Existence et unicité. On va montrer le

ThÉORÈme 3.1. Soit $\Omega$ un ouvert borné régulier de $\mathbb{R}^{n}, n=2$ ou 3 , de frontière $\Gamma$ suffisamment régulière. Supposons que $f$ et $u_{0}$ satisfont

$$
\begin{gathered}
f \in L^{1}\left(0, T ;\left[H^{1}(\Omega)\right]^{n}\right), \\
u_{0} \in V_{3} .
\end{gathered}
$$

Il existe alors $T^{*} \leq \min \left(T, T_{1}\right)$ où

$$
T=T_{1}\left(\Omega, \nu, \alpha,|f|_{L^{1}\left(0, T ;\left[H^{1}(\Omega)\right]^{n}\right)},\left|u_{0}\right|_{V_{3}}\right),
$$

tel que le système (2.3)-(2.4) possède une solution unique $u$ avec les propriétés

$$
\begin{gathered}
u \in L^{\infty}\left(0, T^{*} ; V_{3}\right) \cap C_{W}\left(\left[0, T^{*}\right] ; V_{3}\right) \cap C\left(\left[0, T^{*}\right] ; V\right), \\
u^{\prime} \in L^{2}\left(0, T^{*} ; V\right),
\end{gathered}
$$

où le symbole $C_{W}$ désigne la continuité faible.

Démonstration. La preuve est composée de quatre étapes:

(i) construction de solutions approchées $u_{m}$ de $u$ par la méthode de Galerkin,

(ii) estimations a priori des solutions approchées,

(iii) passage à la limite quand $m \rightarrow+\infty$ par la méthode de compacité,

(iv) unicité.

CAS DE LA DIMENSION 2. (i) Solutions approchées. Soit $W_{m}=\left[w_{1}, \ldots, w_{m}\right]$ l'espace vectoriel engendré par les fonctions propres $w_{1}, \ldots, w_{m}$ définies dans le paragraphe 2. Pour tout $m \in \mathbb{N}^{*}$, fixé, on définit une solution approchée du système (2.3)-(2.4) par

$$
u_{m} \in W_{m}, \quad u_{m}(t)=\sum_{i=1}^{m} g_{i m}(t) w_{i}
$$

et $u_{m}$ solution du système

$$
\begin{gathered}
\left(u_{m}^{\prime}, w_{j}\right)_{V}+\nu\left(\left(u_{m}, w_{j}\right)\right)-b\left(u_{m}, w_{j}, u_{m}-\alpha \Delta u_{m}\right)=\left(f, w_{j}\right), \\
u_{m}(0)=u_{0 m} \in W_{m}, \quad u_{0 m} \rightarrow u_{0} \quad \text { dans } V_{3} \text { si } m \rightarrow+\infty .
\end{gathered}
$$

On obtient ainsi un système de $m$ équations différentielles ordinaires. Des résultats classiques assurent l'existence de $u_{m}$ sur un intervalle $\left[0, T_{m}\right]$. Les estimations a priori qui suivent vont nous permettre de montrer qu'on peut prendre $T_{m}=T_{1}$ où $T_{1}>0$ est indépendant de $m$.

(ii) Estimations a priori. Estimations a priori (I). Multiplions l'équation (3.5) par $g_{j m}$ et sommons sur $j$ de 1 à $m$;

$$
\frac{1}{2} \frac{d}{d t}\left(\left|u_{m}\right|_{V}\right)^{2}+\nu\left\|u_{m}\right\|^{2}-b\left(u_{m}, u_{m}, u_{m}-\alpha \Delta u_{m}\right)=\left(f, u_{m}\right) .
$$


Et grâce aux relations $(2.1)-(2.2)$, on a

$$
\frac{1}{2} \frac{d}{d t}\left(\left|u_{m}\right|_{V}\right)^{2}+\nu\left\|u_{m}\right\|^{2}=\left(f, u_{m}\right) \leq|f|\left|u_{m}\right| .
$$

Mais $\left|u_{m}\right| \leq C_{1}\left|u_{m}\right|_{V}$, où $C_{1}$ est une constante qui dépend seulement de $\Omega$ et $\alpha$; par conséquent

$$
\frac{d}{d t}\left|u_{m}\right|_{V} \leq C_{1}|f|
$$

Des hypothèses (3.1) et (3.6) on déduit finalement que

$$
u_{m} \in \text { borné de } L^{\infty}(0, T ; V) \text {. }
$$

Estimation a priori (II). On réecrit l'équation (3.5) sous la forme

$$
\left(u_{m}^{\prime}, w_{j}\right)_{V}+\left(G\left(u_{m}\right), w_{j}\right)=\left(f, w_{j}\right), \quad 1 \leq j \leq m,
$$

où $G$ est défini par

$$
G\left(u_{m}\right)=-\nu \Delta u_{m}+u_{m} \cdot \nabla\left(u_{m}-\alpha \Delta u_{m}\right) .
$$

Soit $v_{m}$ la solution approchée du problème de Stokes

$$
\begin{gathered}
v_{m}-\alpha \Delta v_{m}+\nabla q_{m}=G\left(u_{m}\right)-f \text { dans } \Omega, \\
\operatorname{div} v_{m}=0 \quad \text { dans } \Omega, \\
v_{m \mid \Gamma}=0 .
\end{gathered}
$$

Comme $\Gamma$ est supposée suffisamment régulière, les solutions $w_{j}$ du problème spectral (2.6) sont aussi suffisamment régulières (par exemple $\left.\left[H^{4}(\Omega)\right]^{2}\right)$. Ainsi pour tout $m$ fixé et $t \in] 0, T[$,

$$
G\left(u_{m}\right) \in\left[H^{1}(\Omega)\right]^{2} .
$$

Utilisant alors des résultats de régularité de Cattabriga (cf. [9]) pour le problème de Stokes, on obtient ainsi $v_{m} \in\left[H^{3}(\Omega)\right]^{2}$. Multiplions maintenant le première équation du système (3.13) par $w_{j}$ et intégrons par parties, il vient que

$$
\left(v_{m}, w_{j}\right)_{v}=\left(G\left(u_{m}\right)-f, w_{j}\right), \quad 1 \leq j \leq m,
$$

de sorte que la relation (3.11) peut s'écrire sous la forme

$$
\left(u_{m}^{\prime}, w_{j}\right)_{V^{\prime}}+\left(v_{m}, w_{j}\right)_{V^{\prime}}=0, \quad 1 \leq j \leq m .
$$

On multiplie maintenant cette dernière relation par $\lambda_{j} g_{j m}$; grâce à (2.6) on a

$$
\left(u_{m}^{\prime}, u_{m}\right)_{V_{3}}+\left(v_{m}, u_{m}\right)_{V_{3}}=0
$$

i.e.,

$$
\begin{aligned}
& \left(u_{m}^{\prime}, u_{m}\right)_{V}+\left(\operatorname{rot}\left(u_{m}^{\prime}-\alpha \Delta u_{m}^{\prime}\right), \operatorname{rot}\left(u_{m}-\alpha \Delta u_{m}\right)\right) \\
& \quad+\left(v_{m}, u_{m}\right)_{v}+\left(\operatorname{rot}\left(v_{m}-\alpha \Delta v_{m}\right), \operatorname{rot}\left(u_{m}-\alpha \Delta u_{m}\right)\right)=0 .
\end{aligned}
$$

Mais étant donnée la régularité de $v_{m}$, on a d'après (3.13)

$$
\operatorname{rot}\left(v_{m}-\alpha \Delta v_{m}\right)=\operatorname{rot}\left[G\left(u_{m}\right)-f\right]:
$$


et grâce à (3.14),

$$
\begin{aligned}
& \left(u_{m}^{\prime}, u_{m}\right)_{V}+\left(\operatorname{rot}\left(u_{m}^{\prime}-\alpha \Delta u_{m}^{\prime}\right), \operatorname{rot}\left(u_{m}-\alpha \Delta u_{m}\right)\right) \\
& \quad+\left(G\left(u_{m}\right)-f, u_{m}\right)+\left(\operatorname{rot}\left[G\left(u_{m}\right)-f\right], \operatorname{rot}\left(u_{m}-\alpha \Delta u_{m}\right)\right)=0 .
\end{aligned}
$$

On observe maintenant que

$$
\begin{gathered}
\left(G\left(u_{m}\right), u_{m}\right)=\nu\left\|u_{m}\right\|^{2}-b\left(u_{m}, u_{m}, u_{m}-\alpha \Delta u_{m}\right)=\nu\left\|u_{m}\right\|^{2} \\
\operatorname{rot} G\left(u_{m}\right)=-\nu \operatorname{rot} \Delta u_{m}+u_{m} \cdot \nabla\left(\operatorname{rot}\left(u_{m}-\alpha \Delta u_{m}\right)\right)-\operatorname{rot} u_{m_{i}} \cdot \nabla\left(u_{m_{i}}-\alpha \Delta u_{m_{i}}\right) .
\end{gathered}
$$

Par conséquent

$$
\begin{aligned}
(\operatorname{rot} & \left.G\left(u_{m}\right), \operatorname{rot}\left(u_{m}-\alpha \Delta u_{m}\right)\right) \\
= & -\nu\left(\operatorname{rot} \Delta u_{m}, \operatorname{rot}\left(u_{m}-\alpha \Delta u_{m}\right)\right)-\left(\operatorname{rot} u_{m_{i}} \cdot \nabla\left(u_{m_{i}}-\alpha \Delta u_{m_{i}}\right), \operatorname{rot}\left(u_{m}-\alpha \Delta u_{m}\right)\right) \\
= & \frac{\nu}{\alpha}\left|\operatorname{rot}\left(u_{m}-\alpha \Delta u_{m}\right)\right|^{2}-\frac{\nu}{\alpha}\left(\operatorname{rot} u_{m}, \operatorname{rot}\left(u_{m}-\alpha \Delta u_{m}\right)\right) \\
& -\left(\operatorname{rot} u_{m_{i}} \cdot \nabla\left(u_{m_{i}}-\alpha \Delta u_{m_{i}}\right), \operatorname{rot}\left(u_{m}-\alpha \Delta u_{m}\right)\right) .
\end{aligned}
$$

Des relations ci-dessus, on déduit de (3.16) que

$$
\begin{aligned}
\frac{1}{2} \frac{d}{d t}\left(\left|u_{m}\right|_{V_{3}}\right)^{2}+\nu\left\|u_{m}\right\|^{2}+\frac{\nu}{\alpha}\left|\omega_{m}-\alpha \Delta \omega_{m}\right|^{2} \\
=\left(f, u_{m}\right)+\left(\operatorname{rot} f, \omega_{m}-\alpha \Delta \omega_{m}\right) \\
\quad+\frac{\nu}{\alpha}\left(\omega_{m}, \omega_{m}-\alpha \Delta \omega_{m}\right)+\left(\operatorname{rot} u_{m_{i}} \cdot \nabla\left(u_{m_{i}}-\alpha \Delta u_{m_{i}}\right), \omega_{m}-\alpha \Delta \omega_{m}\right),
\end{aligned}
$$

où $\omega_{m}=\operatorname{rot} u_{m}$. Utilisant l'inégalité de Young et l'injection $H^{3} \subset W^{1, \infty}$, on a

$$
\frac{d}{d t}\left(\left|u_{m}\right|_{V_{3}}\right)^{2} \leq C_{2}\|f\|_{H^{1}}\left|u_{m}\right|_{V_{3}}+C_{3}\left|u_{m}\right|_{V_{3}}+C_{4}\left(\left|u_{m}\right|_{V_{3}}\right)^{3}
$$

où $C_{2}=C_{2}(\Omega), C_{3}=C_{3}\left(\nu, \alpha, f, u_{0}\right), C_{4}=C_{4}(\Omega)$. Par conséquent,

$$
\frac{d}{d t}\left|u_{m}\right|_{V_{3}} \leq \frac{1}{2} C_{4}\left(\left|u_{m}\right|_{V_{3}}\right)^{2}+g(t)
$$

où $g(t)=\frac{1}{2}\left(C_{2}\|f(t)\|_{H^{1}}+C_{3}\right) \in L^{1}(0, T)$ d'après l'hypothèse (3.1). On déduit ainsi que

$$
\left|u_{m}(t)\right|_{V_{3}} \leq y(t), \quad 0 \leq t \leq T_{1},
$$

où $y(t)$ est la solution de l'équation différentielle

$$
\begin{aligned}
y^{\prime}(t) & =\frac{1}{2} C_{4} y^{2}(t)+g(t), \\
y(0) & =\left|u_{m}(0)\right|_{V_{3}},
\end{aligned}
$$

et $\left(0, T_{1}\right)$ est l'intervalle d'existence de $y(t)$ avec $T_{1}$ dépendant de $C_{4}$ et $g$, c'est à dire de $\nu, \alpha, f, u_{0}$, et $\Omega$.

Utilisant maintenant la convergence (3.6), on déduit de (3.21)

$$
u_{m} \in \text { borné de } L^{\infty}\left(0, T^{*} ; V_{3}\right) \text {, }
$$

où $T^{*}=\min \left(T, T_{1}\right)$. 
Estimation a priori (III). On multiplie la relation (3.15) par $g_{j m}^{\prime}$ et on somme sur $j$ de 1 à $m$

$$
\left(u_{m}^{\prime}, u_{m}^{\prime}\right)_{V}+\left(v_{m}, u_{m}^{\prime}\right)_{V}=0
$$

i.e.,

$$
\left|u_{m}^{\prime}\right|_{V}^{2}=-\left(v_{m}, u_{m}^{\prime}\right)_{V}
$$

Par conséquent,

$$
\left|u_{m}^{\prime}\right|_{V} \leq\left|v_{m}\right|_{V}
$$

Mais,

$$
\left|v_{m}\right|_{V}^{2}=\left(G\left(u_{m}\right)-f, u_{m}\right)=\nu\left\|u_{m}\right\|^{2}-\left(f, u_{m}\right) \leq \nu\left\|u_{m}\right\|^{2}+|f|\left|u_{m}\right|,
$$

d'où grâce à (3.10), $v_{m} \in$ borné de $L^{2}(0, T ; V)$ et donc par (3.23)

$$
u_{m}^{\prime} \in \text { borné de } L^{2}(0, T ; V) \text {. }
$$

(iii) Passage à la limite. Les estimations a priori (3.22) et (3.23) étant vérifiées, on peut donc extraire une sous suite $u_{k}$ telle que lorsque $k \rightarrow+\infty$

$$
\begin{aligned}
& u_{k} \rightarrow u \text { dans } L^{\infty}\left(0, T^{*} ; V_{3}\right) \text { faible étoile, } \\
& u_{k}^{\prime} \rightarrow u^{\prime} \text { dans } L^{2}(0, T ; V) \text { faible. }
\end{aligned}
$$

Utilisant un résultat de compacité (cf. [6] ou [9]), il s'ensuit que

$$
u_{k} \rightarrow u \text { dans } L^{p}\left(0, T^{*} ; W\right) \text { fort } \forall 1<p<+\infty,
$$

et, en particulier, $u_{k} \rightarrow u$ p.p. dans $Q_{T^{*}}$.

Avec ces convergences, il est maintenant facile de voir que $u$ vérifie le système (2.3)-(2.4).

Remarque 3.1. Puisque $u \in L^{\infty}\left(0, T^{*} ; V\right)$ et $u^{\prime} \in L^{\infty}\left(0, T^{*} ; V\right)$, donc $u \in$ $C\left(\left[0, T^{*}\right] ; V\right)$. De plus comme $u \in L^{\infty}\left(0, T^{*} ; V_{3}\right)$, par conséquent $u \in$ $C_{W}\left(\left[0, T^{*}\right] ; V_{3}\right)$.

Unicité. Soient $u_{1}$ et $u_{2}$ deux solutions du problème (2.3)-(2.4) avec la même donnée initiale. Posant $u=u_{1}-u_{2}$, alors $u(0)=0$ et

$$
\begin{aligned}
& \frac{1}{2} \frac{d}{d t}\left(|u|_{V}\right)^{2}+\nu\|u\|^{2}-b(u, u, u-\alpha \Delta u) \\
& \quad-b\left(u, u, u_{2}-\alpha \Delta u_{2}\right)-b\left(u_{2}, u, u-\alpha \Delta u\right)=0
\end{aligned}
$$

c'est à dire que grâce aux relations (2.1)-(2.2),

$$
\frac{1}{2} \frac{d}{d t}\left(|u|_{V}\right)^{2}+\nu\|u\|^{2}=b\left(u, u, u_{2}-\alpha \Delta u_{2}\right)-b\left(u_{2}, u, u-\alpha \Delta u\right) .
$$

Il est facile de voir que puisque $H^{1} \subset L^{4}$, l'inégalité de Hölder entraîne que

$$
\left|b\left(u, u, u_{2}-\alpha \Delta u_{2}\right)\right| \leq C_{1}\left|u_{2}\right|_{V_{3}}\left(|u|_{V}\right)^{2} .
$$

Pour estimer le second terme, on remarque que puisque $H^{3} \subset W^{1, \infty}$,

$$
\left|b\left(u_{2}, u, \Delta u\right)\right|=\left|b\left(\partial_{k} u_{2}, u, \partial_{k} u\right)\right| \leq C_{2}\left|u_{2}\right|_{V_{3}}\left(|u|_{V}\right)^{2} .
$$


Appliquons ces estimations dans la relation (3.25), il vient que

$$
\frac{1}{2} \frac{d}{d t}\left(|u|_{V}\right)^{2} \leq C_{3}\left|u_{2}\right|_{V_{3}}\left(|u|_{V}\right)^{2} .
$$

Comme $u_{2} \in L^{\infty}\left(0, T^{*} ; V_{3}\right)$, le lemme de Gronwall entraîne que

$$
\left(|u|_{V}\right)^{2} \leq 0 \quad \forall t \in\left[0, T^{*}\right]
$$

i.e., $u_{1}=u_{2}$.

CAS DE LA DIMENSION 3. (i) Solutions approchées. On reprend le problème (3.5)(3.6) sans aucun changement.

(ii) Estimations a priori. Estimation a priori (I). La propriété (2.2) n'étant plus vérifiée, on ne peut obtenir directement l'estimation (3.10) (celle-ci n'était en fait pas fondamentale puisque elle est pratiquement contenue dans l'estimation (3.22)).

On réecrit à nouveau l'équation (3.5) sous la forme

$$
\left(u_{m}^{\prime}, w_{j}\right)_{V}+\left(G\left(u_{m}\right), w_{j}\right)=\left(f, w_{j}\right), \quad 1 \leq j \leq m,
$$

où $G$ a été défini par (3.12). On considère ici encore le problème de Stokes (3.13) et l'on aboutit comme dans le cas bidimensionnel à la relation (cf. (3.16))

$$
\begin{aligned}
& \left(u_{m}^{\prime}, u_{m}\right)_{V}+\left(\operatorname{rot}\left(u_{m}^{\prime}-\alpha \Delta u_{m}^{\prime}\right), \operatorname{rot}\left(u_{m}-\alpha \Delta u_{m}\right)\right) \\
& \quad+\left(v_{m}, u_{m}\right)_{V}+\left(\operatorname{rot}\left(v_{m}-\alpha \Delta v_{m}\right), \operatorname{rot}\left(u_{m}-\alpha \Delta u_{m}\right)\right)=0
\end{aligned}
$$

puis,

$$
\begin{aligned}
& \left(u_{m}^{\prime}, u_{m}\right)_{V}+\left(\operatorname{rot}\left(u_{m}^{\prime}-\alpha \Delta u_{m}^{\prime}\right), \operatorname{rot}\left(u_{m}-\alpha \Delta u_{m}\right)\right) \\
& \quad+\left(G\left(u_{m}\right)-f, u_{m}\right)+\left(\operatorname{rot}\left[G\left(u_{m}\right)-f\right], \operatorname{rot}\left(u_{m}-\alpha \Delta u_{m}\right)\right)=0
\end{aligned}
$$

On note alors que

$$
\begin{aligned}
\left(G\left(u_{m}\right), u_{m}\right)= & \nu\left\|u_{m}\right\|^{2}-b\left(u_{m}, u_{m}, u_{m}-\alpha \Delta u_{m}\right) \\
= & \nu\left\|u_{m}\right\|^{2}+\alpha b\left(u_{m}, u_{m}, \Delta u_{m}\right), \\
\operatorname{rot} G\left(u_{m}\right)= & -\nu \operatorname{rot} \Delta u_{m}+u_{m} \cdot \nabla\left(\operatorname{rot}\left(u_{m}-\alpha \Delta u_{m}\right)\right)+\nabla u_{m_{j}} \wedge \nabla\left(u_{m_{j}}-\alpha \Delta u_{m_{j}}\right) \\
& -\nabla u_{m} \cdot \operatorname{rot}\left(u_{m}-\alpha \Delta u_{m}\right) .
\end{aligned}
$$

Par conséquent,

$$
\begin{aligned}
\left(\operatorname{rot} G\left(u_{m}\right), \operatorname{rot}\left(u_{m}-\alpha \Delta u_{m}\right)\right)= & -\nu\left(\operatorname{rot} \Delta u_{m}, \operatorname{rot}\left(u_{m}-\alpha \Delta u_{m}\right)\right) \\
& -\left(\nabla u_{m} \cdot \operatorname{rot}\left(u_{m}-\alpha \Delta u_{m}\right), \operatorname{rot}\left(u_{m}-\alpha \Delta u_{m}\right)\right) \\
& +\left(\nabla u_{m_{j}} \wedge \nabla\left(u_{m_{j}}-\alpha \Delta u_{m_{j}}\right), \operatorname{rot}\left(u_{m}-\alpha \Delta u_{m}\right)\right) \\
= & \frac{\nu}{\alpha}\left|\operatorname{rot}\left(u_{m}-\alpha \Delta u_{m}\right)\right|^{2}-\frac{\nu}{\alpha}\left(\operatorname{rot} u_{m}, \operatorname{rot}\left(u_{m}-\alpha \Delta u_{m}\right)\right) \\
& -\left(\nabla u_{m} \cdot \operatorname{rot}\left(u_{m}-\alpha \Delta u_{m}\right), \operatorname{rot}\left(u_{m}-\alpha \Delta u_{m}\right)\right) \\
& +\left(\nabla u_{m_{j}} \wedge \nabla\left(u_{m_{j}}-\alpha \Delta u_{m_{j}}\right), \operatorname{rot}\left(u_{m}-\alpha \Delta u_{m}\right)\right)
\end{aligned}
$$

L'injection $H^{3} \subset W^{1, \infty}$ ayant encore lieu, les mêmes techniques qu'en dimension 2 permettent d'obtenir l'estimation (cf. (3.22))

$$
u_{m} \in \text { borné de } L^{\infty}\left(0, T^{*} ; V_{3}\right) .
$$


Estimation a priori (II). Comme pour l'équation (3.23), on a $\left|u_{m}^{\prime}\right|_{V} \leq\left|v_{m}\right|_{V}$, où

$$
\begin{aligned}
\left|v_{m}\right|_{V}^{2} & =\nu\left\|u_{m}\right\|^{2}+\alpha b\left(u_{m}, u_{m}, \Delta u_{m}\right)-\left(f, u_{m}\right) \\
& \leq \nu\left\|u_{m}\right\|^{2}+|f|\left|u_{m}\right|+C\left(\left\|u_{m}\right\|_{V_{3}}\right)^{3},
\end{aligned}
$$

où la constante $C>0$ dépend de $\Omega$ et $\alpha$. Par conséquent on a

$$
u_{m}^{\prime} \in \text { borné de } L^{2}(0, T ; V) \text {. }
$$

(iii) Passage à la limite. Il se fait de façon similaire à la dimension 2.

Enfin la remarque 3.1 et l'unicité sont encore valables.

4. Autres formulations du problème. L'objet de ce paragraphe est de donner d'autres formulations du problème (1.5)-(1.8) ainsi q'un critère d'unicité.

Dans ce qui précéde, on a pris (cf. (2.5)) $V$ comme espace pivot. Ici l'espace $H$ sera identifié à $H^{\prime}$ et servira de nouveau pivot dans les injections continues et denses

$$
W \subset V \subset H \subset V^{\prime} \subset W^{\prime},
$$

où, de plus, les deux premières injections sont compactes.

On définit la forme trilinéaire

$$
\tilde{b}(u, v, w)=b(u, v, w-\alpha \Delta w) .
$$

LEMME 4.1. La forme trilinéaire $(u, v, w) \rightarrow \tilde{b}(u, v, w)$ est continue sur $W \times V \times$ $W$.

Démonstration.

$$
|\tilde{b}(u, v, w)| \leq\|u\|_{L^{\infty}}\|v\||w-\alpha \Delta w| \leq C\|u\|_{2}\|v\|\|w\|_{2},
$$

puisque l'injection $W \subset\left[L^{\infty}(\Omega)\right]^{n}$ est continue si $n \leq 3$ (on rappelle que $\|\cdot\|$ désigne la norme dans $\left[H_{0}^{1}(\Omega)\right]^{n}, V$ sera muni de cette norme).

On définit maintenant les notations suivantes. Pour $u, v \in W$, on note $B(u, v)$ l'élément de $V^{\prime}$ défini par

$$
\langle B(u, v), w\rangle=\tilde{b}(u, v, w) \quad \forall w \in V,
$$

et on pose

$$
B u=B(u, u) \in V^{\prime} \subset W^{\prime} \quad \forall u \in W .
$$

On peut maintenant poser

Problème 1. On donne

$$
\begin{gathered}
f \in L^{2}\left(0, T ; W^{\prime}\right), \\
u_{0} \in W .
\end{gathered}
$$

On cherche $u, p \in \mathscr{D}^{\prime}\left(Q_{T}\right)$ tels que

$$
\begin{gathered}
u \in L^{\infty}(0, T ; W), \\
u^{\prime}-\alpha \Delta u^{\prime}-\nu \Delta u+(u \cdot \nabla)(u-\alpha \Delta u)+\nabla p=f \quad \text { dans } Q_{T}, \\
u(0)=u_{0} \quad \operatorname{dans} \Omega .
\end{gathered}
$$


Remarque 4.1. Malgré la précision de l'énoncé de ce problème, on n'a de renseignements ni sur $u^{\prime}$ ni su $p$ autre que la relation (4.8); ce qui pose le problème du sens à donner à (4.9).

Soit alors $v \in \mathscr{V}$, d'après $(4.8)$ on a

$$
\left\langle u^{\prime}-\alpha \Delta u^{\prime}, v\right\rangle=-\nu((u, v))+b(u, v, u-\alpha \Delta u)+\langle f, v\rangle .
$$

Mais les inégalités suivantes

$$
\begin{aligned}
|((u, v))| \leq\|u\|_{2}|v| & \leq C\|u\|_{2}\|v\| \leq C\|u\|_{2}\|v\|_{2}, \\
|b(u, v, u-\alpha \Delta u)| & \leq\|u\|_{2}^{2}\|v\| \leq C\|u\|_{2}^{2}\|v\|_{2}, \\
|\langle f, v\rangle| & \leq\|f\|_{W^{\prime}}\|v\|_{2},
\end{aligned}
$$

montrent que

$$
A_{1} u^{\prime} \in L^{2}\left(0, T ; W^{\prime}\right),
$$

où $A_{1}$ désigne l'opérateur défini par

$$
\left\langle A_{1} w, v\right\rangle=(w, v)+\alpha((w, v)) \quad \forall v, w \in V .
$$

De plus, comme l'opérateur $A_{1}: W \rightarrow H$ est un isomorphisme, alors

$$
u^{\prime} \in L^{2}(0, T ; H) \text {. }
$$

Ainsi l'équation (4.9) a un sens (dans $H$ ).

Remarque 4.2. On peut améliorer la régularité de $u^{\prime}$ dans l'équation (4.16) de la façon suivante. On suppose que $f$ vérifie

$$
f \in L^{2}\left(0, T ; V^{\prime}\right) \text {. }
$$

En réecrivant l'équation (4.13) sous la forme $|\langle f, v\rangle| \leq\|f\|_{V^{\prime}}\|v\|$, on déduit des inégalités $(4.11)$ et $(4.12)$ que

$$
A_{1} u^{\prime} \in L^{2}\left(0, T ; V^{\prime}\right)
$$

et comme $A_{1}: V \rightarrow V^{\prime}$ est un isomorphisme, donc

$$
u^{\prime} \in L^{2}(0, T ; V) \text {. }
$$

La remarque 4.2 nous conduit à une autre formulation du problème 1 où maintenant $f$ vérifie la condition (4.17).

Problème 2. Sous les hypothèses (4.5) et (4.17), on cherche $u$ tel que

$$
\begin{gathered}
u \in L^{\infty}(0, T ; W), \\
\left\langle u^{\prime}-\alpha \Delta u^{\prime}, v\right\rangle+\nu((u, v))-b(u, v, u-\alpha \Delta u)=\langle f, v\rangle \quad \forall v \in W, \\
u(0)=u_{0} .
\end{gathered}
$$

Montrons l'équivalence de ces deux problèmes.

Soit $u$ solution du problème 1 , alors on a l'équation (4.10) $\forall v \in \mathscr{V}$. Soit maintenant $v \in W$; il existe une suite $v_{n} \in \mathscr{V}$ telle que $v_{n} \rightarrow v$ dans $V$, et vérifiant

$$
\left\langle A_{1} u^{\prime}, v_{n}\right\rangle+\nu\left(\left(u, v_{n}\right)\right)-b\left(u, v_{n}, u-\alpha \Delta u\right)=\left\langle f, v_{n}\right\rangle \text {. }
$$


Le passage à la limite montre alors que $u$ est solution du problème 2 .

Réciproquement soit $u$ une solution du problème 2; posons

$$
u^{\prime}-\alpha \Delta u^{\prime}-\nu \Delta u+(u \cdot \nabla)(u-\alpha \Delta u)-f=S,
$$

alors $S \in\left[\mathscr{D}^{\prime}\left(Q_{T}\right)\right]^{n}$ et

$$
\langle S, v\rangle=0 \quad \text { dans } \quad\left[\mathscr{D}^{\prime}(0, T)\right]^{n} \quad \forall v \in \mathscr{V} .
$$

D'où, d'après le théorème de De Rham (cf. [4] ou [5]), $S=-\nabla p$ pour une certaine distribution $p \in \mathscr{D}^{\prime}\left(Q_{T}\right)$. Ceci achève de montrer l'équivalence des problèmes 1 et 2.

Le résultat suivant donne quelques propriétés des solutions du problème 2 .

Proposition 4.1. Toute solution du problème 2 vérifie:

$$
u^{\prime} \in L^{2}(0, T ; V) \text {, }
$$

$u$ est p.p. égale à une fonction continue de $[0, T]$ dans $V$,

$u \in C_{W}([0, T] ; W)$, i.e., $u$ est faiblement continue de $[0, T]$ dans $W$.

Avant d'établir cette proposition, on a besoin du lemme suivant (cf. [9]).

Lemme 4.2. Soient $X$ et $Y$ deux Banach tels que $X \subset Y$ avec injection continue. Si une fonction $g \in L^{\infty}(0, T ; X)$ est faiblement continue à valeurs dans $Y$, alors $g$ est faiblement continue à valeurs dans $X$.

Démonstration de la proposition 4.1. La condition (4.24) est satisfaite par la remarque 4.2. Comme de plus,

$$
u \in L^{\infty}(0, T ; W) \subset L^{2}(0, T ; V),
$$

la propriété (4.25) suit immédiatement. Appliquant le lemme 4.2 et la propriété (4.25), alors (4.26) en résulte.

THÉORÈmE 4.3. Le problème 2 possède au plus une solution dans la classe

$$
L^{\infty}(0, T ; W) \cap L^{1}\left(0, T ;\left[H^{s}(\Omega)\right]^{n}\right),
$$

pour $s>1+n / 2$.

Démonstration. Soient $u$ et $u^{*}$ deux solutions du problème 2; posons $w=u-u^{*}$. En faisant la différence entre les équations (4.2) pour $u$ et $u^{*}$, on a

$\left\langle w^{\prime}-\alpha \Delta w^{\prime}, v\right\rangle+\nu((w, v))+b\left(u^{*}, v, u^{*}-\alpha \Delta u^{*}\right)-b(u, v, u-\alpha \Delta u)=0 \quad \forall v \in V$.

Prenons $v=w$ et notons que

$$
\left\langle w^{\prime}-\alpha \Delta w^{\prime}, w\right\rangle-\left\langle A_{1} w^{\prime}, w\right\rangle=\left(w^{\prime}, w\right)+\alpha\left(\left(u^{\prime}, w\right)\right),
$$

puisque $w$ et $w^{\prime} \in L^{2}(0, T ; V)$. Par conséquent, on a

$$
\left\langle w^{\prime}-\alpha \Delta w^{\prime}, w\right\rangle=\frac{1}{2} \frac{d}{d t}\left(|w(t)|^{2}+\alpha\|w(t)\|^{2}\right) .
$$


Par ailleurs,

$$
\begin{aligned}
& \left|b(u, w, u-\alpha \Delta u)-b\left(u^{*}, w, u^{*}-\alpha \Delta u^{*}\right)\right| \\
& \quad=\left|b(w, w, u-\alpha \Delta u)-b\left(u^{*}, w, w-\alpha \Delta w\right)\right| .
\end{aligned}
$$

Utilisant l'inégalité de Hölder, on a

$$
|b(w, w, u-\alpha \Delta u)| \leq\|w\|_{L^{p}}\|w\||u-\alpha \Delta u|_{L^{q}}, \quad \frac{1}{p}+\frac{1}{q}=\frac{1}{2} .
$$

Si $n=2, q>2$ équivaut à $p<+\infty$; et $H^{1} \subset L^{p} \forall p<+\infty$. De plus, comme $W^{r, 2} \subset W^{2, q}$ avec $r=3-(2 / q)$; alors $q>2$ équivaut à $r>2$, i.e., l'inégalité (4.30) s'écrit

$$
|b(w, w, u-\alpha \Delta u)| \leq\|u\|_{H^{s}}\|w\|^{2}, \quad s>2 .
$$

Si $n=3$ en utilisant les injections convenables, on montre un inégalité du type (4.31) avec $s>5 / 2$.

Par ailleurs,

où $s>1+(n / 2)$.

$$
\begin{aligned}
\left|b\left(u^{*}, w, w-\alpha \Delta w\right)\right| & =\alpha\left|b\left(\partial_{k} u^{*}, w, \partial_{k} w\right)\right| \\
& \leq \alpha C_{k}\left\|\partial_{k} u^{*}\right\|_{L^{\infty}}\|w\|^{2} \\
& \leq C\left\|u^{*}\right\|_{H^{s}}\|w\|^{2}
\end{aligned}
$$

Finalement, en reportant les relations (4.28)-(4.32) dans l'égalité (4.27), on obtient

$$
\frac{1}{2} \frac{d}{d t}\left(|w(t)|^{2}+\|w(t)\|^{2}\right)+\nu\|w(t)\|^{2} \leq C\left(\|u(t)\|_{H^{s}}+\left\|u^{*}(t)\right\|_{H^{s}}\right)\|w(t)\|^{2},
$$

de sorte que grâce à l'hypothèse, la fonction $t \rightarrow\|u(t)\|_{H^{s}}+\left\|u^{*}(t)\right\|_{H^{s}}$ appartient à $L^{1}(0, T)$ et l'utilisation du lemme de Gronwall donne $w=0$, i.e., l'unicité.

\section{BIBLIOGRAPHIE}

[1] C. Amrouche, Thèse de troisième cycle: Etude globale des fluides de troisième grade, Université Paris 6 (1986)

[2] C. Amrouche et V. Girault, Une méthode d'approximation mixte des équations de fluides non newtoniens de troisieme grade, Numerische Mathematik, vol. 53, Springer-Verlag, Berlin, 1988 , pp. 315-349

[3] D. Cioranescu and E. H. Ouazar, Existence and uniqueness for fluids of second grade, Non Linear Partial Differential Equations, vol. 109, Collège de France Seminar, Pitman, New York, 1983, pp. $178-197$

[4] G. de Rham, Variétés Différentiables, Hermann, Paris, 1960

[5] V. Girault and P. A. Raviart, Finite Element Methods for Navier-Stokes Equations, Springer-Verlag, Berlin, 1986

[6] J. L. Lions, Quelques Méthodes de Résolution des Problèmes aux Limites non Linéaires, GauthierVillars, Paris, 1969

[7] P. A. Oskolkov, The uniqueness and global solvability of boundary-value problems for the equations of motion for aqueous solutions of polymers, Institua im. V. A. Steklova an SSSR, Leningrad, vol. 38, 1973, pp. $98-136$

[8] P. A. Oskolkov, Solvability in the large of the first boundary-value problem for a quasilinear thirdorder system pertaining to the motion of a viscous fluid, Instituta im V. A. Steklova Akad. Nauk SSSR, Leningrad, vol. 27, 1972, pp. 145-160

[9] R. Temam, Theory and Numerical Analysis of the Navier-Stokes Equations, North-Holland, Amsterdam, 1977 